\title{
Effect of Diluent on Molecular Motion and Glass Transition in Polymers. V. The System Poly(methyl methacrylate)/Toluene
}

\author{
Keiichiro ADACHI and Tadao KoTAKA \\ Department of Macromolecular Science, Faculty of Science, \\ Osaka University, Toyonaka, Osaka 560, Japan.
}

(Received November 30, 1980)

\begin{abstract}
Dielectric and thermal behavior of toluene solutions of an isotactic-rich and a syndiotactic-rich poly(methyl methacrylate) have been investigated over wide ranges of concentration and temperature to examine the effect of stereoregularity. Three dielectric relaxations designated as $\alpha, \beta$, and $\gamma$ were observed in both systems. These processes can be assigned, respectively, to the process due to segmental motion of the polymer chains, rotation of toluene molecules, and secondary motion of toluene coupled with those of the polymer. For both systems, plots of loss maximum frequency versus reciprocal temperature for the $\alpha$ process conform to the Vogel-Tamman equation. The Vogel-Tamman parameter $B$ for solutions of the syndiotactic polymer is larger than that for the isotactic polymer, while the other two parameters $A$ and $T_{0}$ are nearly independent of stereoregularity. Differential thermal analysis curves for solutions with low to medium concentrations each exhibits a two-step change, which can be assigned to the two glass. transitions, $T_{\mathrm{gl}}$ and $T_{\mathrm{gII}}$. The $T_{\mathrm{gl}}$ for the solutions of the syndiotactic polymer is higher than that of the isotactic polymer, while the $T_{\mathrm{gll}}$ is the same for both polymers.

KEY WORDS Poly(methyl methacrylate) / Dielectric Relaxation / Glass

Transition / Stereoregularity / Diluent Effect /
\end{abstract}

Since Fox et al. reported properties of stereoregular poly(methyl methacrylate)s (PMMA), ${ }^{1}$ many investigations have been carried out on the influence of stereoregularity on dielectric and mechanical relaxations in bulk PMMAs. ${ }^{2-5}$ Mikhailov et al. showed that dielectric loss versus temperature curves for isotactic and syndiotactic PMMAs are markedly diffrent from each other in relaxation strength and loss maximum temperature for the primary and secondary processes. ${ }^{3}$ The glass transition temperature $T_{\mathrm{g}}$ also depends strongly on the stereoregularity, i.e., the difference in $T_{\mathrm{g}}$ between isotactic and syndiotactic PMMAs is about $80 \mathrm{~K}^{6}$ Dielectric measurements on solutions of radically polymerized PMMAs have also been made by several authors. ${ }^{8-12}$ Mashimo et al. studied solutions of a PMMA over a wide range of concentration at a fixed temperature. ${ }^{9}$ However, the range of concentration studied by other authors was limited to less than $20 \%$. A few studies have been carried out on the effect of stereoregularity on the dielectric properties of PMMA-diluent systems in a limited range of concentration. ${ }^{7}$ Therefore it is of interest to investigate these systems in the whole range of concentrations from pure solvent to bulk polymer.

In this study, we have carried out dielectric measurements and differential thermal analysis on toluene solutions of two PMMAs with different stereoregularity. Their dielectric behavior is compared on the same principle of analysis as was used in the previous studies on other polymer/diluent systems. ${ }^{13-16}$

\section{EXPERIMENTAL}

Two samples of PMMA were used. One was a commercial sample and used after purification by fractional precipitation. We designate this polymer as PMMA-S. The other was prepared by anionic polymerization at $230 \mathrm{~K}$ in toluene (TOL) with $n$ butyllithium as an initiator, and is termed PMMAI. Viscosity-average molecular weights of PMMA-S and PMMA-I were $1.9 \times 10^{5}$ and $5.4 \times 10^{5}$, re- 
spectively. Stereoregularity of these samples was determined by high resolution proton magnetic resonance (NMR) spectroscopy. Measurement of NMR was made at $100 \mathrm{MHz}$ on a Varian XL-100 spectrometer. The stereoregularity defined as the content of isotactic dyads $\psi$ was determined by comparing three lines due to $\alpha$ methyl resonance and using the relation,

$$
\Psi=(i+h / 2) /(i+h+s)
$$

where $i, h$, and $s$ denote, respectively, the heights of the lines due to isotactic, heterotactic, and syndiotactic triad sequences. ${ }^{17}$ The values of $\Psi$ thus determined for PMMA-I and PMMA-S were 0.78 and 0.27 , respectively. The solvent toluene (TOL) was dried by refluxing it over sodium wire, and distilled just before use.

Dielectric measurements between $3.1 \mathrm{~Hz}$ and $1 \mathrm{MHz}$ was made on a capacitance bridge (General Radio 1615-A), an automatic bridge (YokogawaHewlett-Packard 4270-A) and a Cole-Gross type bridge (Showa Denki). Differential thermal analysis (DTA) was performed at a heating rate of $5 \mathrm{~K}$ $\min ^{-1}$. Details of the measurements have been described previously. ${ }^{13}$

\section{RESULTS AND DISCUSSION}

Representative dielectric loss factor $\varepsilon^{\prime \prime}$ versus temperature $T$ curves are shown in Figure 1 for solutions of PMMA-I and PMMA-S with concentrations of 30 and $27 \%$ by weight, respectively. Three loss peaks are seen in the figure, as observed in the studies on other systems. ${ }^{13-16}$ We designate these relaxations as $\alpha, \beta$, and $\gamma$ in the order of decreasing peak temperature. The marked difference in the dielectric behavior of bulk PMMAs is not seen in the solution state. In Figure 1, we recognize that the loss maximum $\varepsilon_{\max }^{\prime \prime}$ for the $\alpha$ process of the PMMA-S solution is slightly larger than that of PMMA-I and that the former exhibits the $\alpha$ peak temperature slightly higher than the latter.

Figure 2 shows the concentration dependence of the loss peak temperatures at $1 \mathrm{kHz}$. For each of the bulk polymers, two maximum temperatures are seen in Figure 2. The one at the higher temperature is due to the primary $\alpha$ relaxation, and the other at the lower temperature is due to the rotation of the side groups. ${ }^{2}$ We denote this secondary relaxation of the bulk polymer by $\beta^{\prime}$ to distinguish it from the $\beta$ process found in the toluene solutions. On the other hand, for pure toluene, two loss peaks corresponding to the primary and secondary processes of toluene molecules are found, and we designate these as the $\alpha_{0}$ and $\beta_{0}$ processes. Based on these data, the mechanisms of the molecular motions for the $\alpha, \beta$, and $\gamma$ processes may be assigned as follows.

We observed that with increasing polymer concentration the height of the $\alpha$ peak increased, while that of the $\beta$ peak decreased rather rapidly and disappeared in the range of concentration above $50 \%$. The height of the $\gamma$ peak also decreased rather gradually, and the $\gamma$ peak persisted until the polymer concentration exceeded $90 \%$. These results suggest that the $\alpha$ process is associated with the

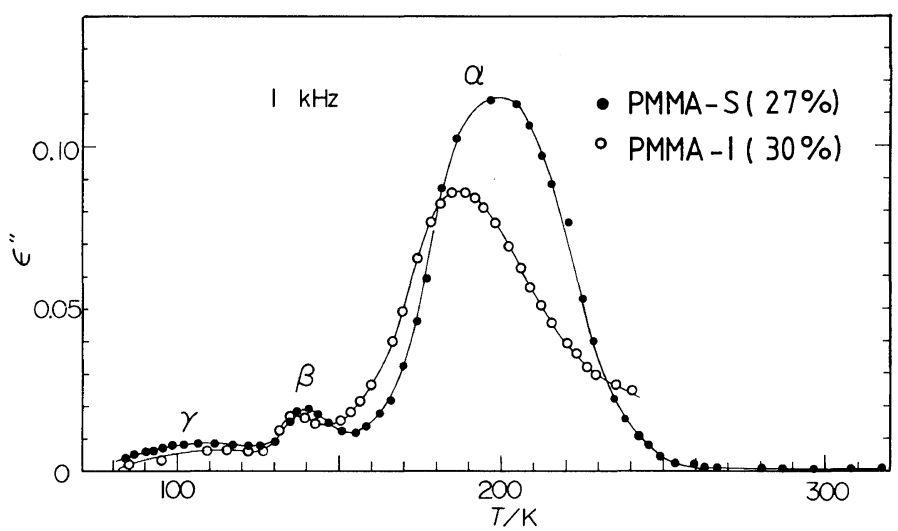

Figure 1. Temperature dependence of dielectric loss factor $\varepsilon^{\prime \prime}$ for the toluene solution of PMMA-I with concentration of $30.0 \%$ and that of PMMA-S with concentration of $26.7 \%$. 


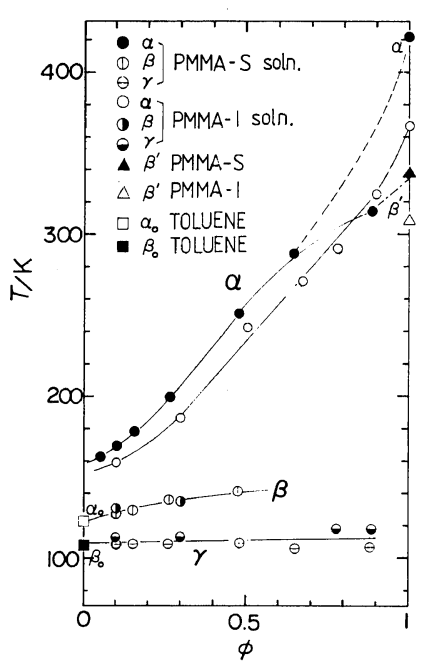

Figure 2. Loss peak temperatures for the $\alpha, \beta$, and $\gamma$ processes measured at $1 \mathrm{kHz}$ versus weight fraction $\phi$ of the PMMAs.

motions of PMMA molecules, while the $\beta$ and $\gamma$ processes are related with the motions of toluene molecules.

As can be seen in Figure 2, the $\alpha$ peak temperature for PMMA-I solutions can be extrapolated to the primary loss peak temperature of bulk PMMA-I. Similarly, the $\alpha$ peak temperature for PMMA-S solutions with concentration $\phi$ below $70 \%$ appears to be extrapolated to the primary loss peak temperature of bulk PMMA-S, as indicated by a dashed curve in Figure 2. These results imply that the $\alpha$ processes for these polymer solutions are due to the main-chain segmental motions. However, the $\alpha$ peak temperature for the $89 \%$ PMMA-S solution is found far below the dashed curve, and it appears to merge with the $\beta^{\prime}$ peak for the bulk polymer, as shown by a dot-dashed line in Figure 2. In other words, the addition of a small amount of toluene to bulk PMMA-S appreciably lowers the $\alpha$ peak temperature of the bulk polymer and the $\alpha$ process is overwhelmed by the $\beta^{\prime}$ process. With the further addition of toluene to decrease $\phi$ below $70 \%$, the $\alpha$ peak temperature for the main-chain motions is markedly reduced and, at the same time, the $\beta^{\prime}$ process is now overwhelmed by the $\alpha$ process. This behavior is similar to that observed in the previous study on the poly(methyl acrylate)/toluene system (PMA/TOL) ${ }^{16}$ Hence the previous discussion on this system also applies to the present PMMA/TOL

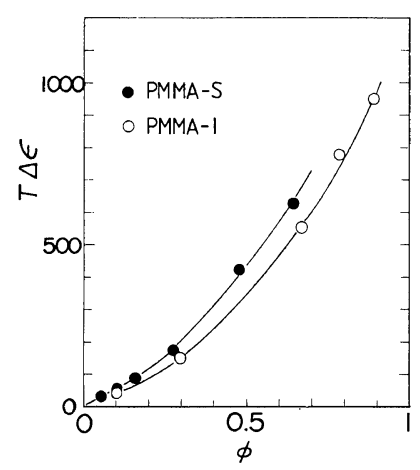

Figure 3. Magnitude of dielectric dispersion $T \Delta \varepsilon$ for the $\alpha$ process versus weight fraction $\phi$ of the PMMAs.

system.

When the relaxation time for the rotation of side groups is shorter than that for the main-chain segmental motion, these processes give rise to two separated loss peaks. On the contrary, when the side group motion is slower than the segmental motions, the former becomes inactive in the dielectric spectrum. ${ }^{9.16}$ Since diluent molecules may act as a plasticizer, the rate of the main-chain motions increases rapidly, but that of the side-group motion is hardly affected. Therefore, a transition occurs at a certain concentration from a state in which the relaxation time for the main-chain motions is greater than that for the side group motion to a state where this relation is reversed. From Figure 2, we estimate the critical concentration to be about $70 \%$ for the PMMA-S solutions. In the PMMA-S solution with a concentration of $89 \%$, two loss peaks due to the $\alpha$ and $\beta^{\prime}$ processes still overlap and are not observed separately, since the $\varepsilon_{\max }^{\prime \prime}$ for the $\alpha$ process is much smaller than that of the $\beta^{\prime}$ process. ${ }^{2,3}$

The $\beta$ and $\gamma$ processes for toluene solutions of both PMMAs correspond, respectively, to the primary $\left(\alpha_{0}\right)$ and the secondary $\left(\beta_{0}\right)$ processes of pure toluene, as can be seen in Figure 2. The $\alpha_{0}$ and $\beta_{0}$ processes of toluene can be ascribed, respectively, to the overall rotation and the so-called local motion ${ }^{2}$ of its molecules. The same mechanisms must apply to the $\beta$ and $\gamma$ processes. However, we cannot assess the extent to which the motion of PMMA molecules is related to these relxation processes. In a previous study on the system of polystyrene (PS) and toluene, it was confirmed from broad line NMR mesurements that the $\gamma$ process in this system is due to a 
local motion of toluene molecules coupled with that of PS. The similarity in dielectric behavior for the $\gamma$ processes between the PMMA/TOL and PS/TOL systems allows us to speculate that the $\gamma$ process in the PMMA/TOL system is also due to the same mechanism.

The magnitude of dielectric dispersion, $\Delta \varepsilon=$ $\varepsilon_{0}-\varepsilon_{\infty}$, for the $\alpha$ process of each solution was determined from Cole-Cole plots. ${ }^{2}$ Here, $\varepsilon_{0}$ and $\varepsilon_{\infty}$ are static and high frequency dielectric constants, respectively. In Figure 3, the results are plotted in the form of $T \Delta \varepsilon v s$. $\phi$ in order to compensate for the effect of temperature $T$ on $\Delta \varepsilon$. As is seen in Figure 3, $T \Delta \varepsilon$ of PMMA-S solution is always larger than that of the PMMA-I solution with the corresponding concentration. Since the chemical composition of both polymers is the same, this difference must be attributed to the difference in the stereoregularity, which results in the difference in the orientational correlation between dipoles. If there is a correlation between neighboring dipoles, the $\Delta \varepsilon$ is given by the Onsager-Kirkwood-Fröhlich equation, ${ }^{2,18}$

$$
\Delta \varepsilon=\frac{4 \pi N \mu^{2} g \varepsilon_{0}\left(\varepsilon_{\infty}+2\right)^{2}}{9 k T\left(2 \varepsilon_{0}+\varepsilon_{\infty}\right)}
$$

where $N$ is the number of dipoles with the moment $\mu$ in unit volume and $g$ is the Kirkwood correlation parameter. As is well known, the value of $g$ is larger than unity when dipoles tend to orient parallel with one another and smaller than unity when they orient anti-parallel. The value of $g$ for the $67 \%$ PMMA-I solution and that for the $65 \%$ PMMA-S solution have been calculated to be 0.58 and 0.67 , respectively. In this calculation, we used $1.67 \mathrm{D}$ for the dipole moment of the monomer unit. ${ }^{19}$ Since the average distance between neighboring dipoles in the

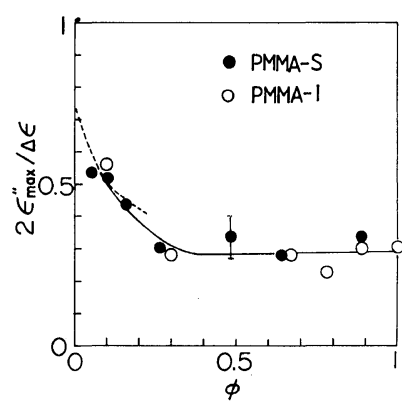

Figure 4. Plots of $2 \varepsilon_{\max }^{\prime \prime} / \Delta \varepsilon$ with respect to weight fraction $\phi$ of the PMMAs. ----, data by Phillips and Singh (ref 10). molecule of PMMA-S is larger than that of PMMA-I, the interaction and therefore the correlation between the dipoles in PMMA-S is weaker than that between those in PMMA-I.

One of the measures of the distribution of dielectric relaxation time is the quantity $2 \varepsilon_{\max }^{\prime \prime} / \Delta \varepsilon$. As is well known, this 'value is unity for a dispersion of the Debye type with a single relaxation time, and decreases as the distribution becomes broader. Figure 4 shows the concentration dependence of $2 \varepsilon_{\max }^{\prime \prime} / \Delta \varepsilon$ for the $\alpha$ relaxation process. As was pointed out by Phillips and Singh, ${ }^{10}$ this value rapidly decreases in the range of low concentration, but soon levels off above a $30 \%$ concentration, which implies that the distribution of relaxation time is independent of concentration in the range above $30 \%$. As can be seen in Figure 3, the breadth of the distribution of relaxation time is also independent of stereoregularity.

Figure 5 shows the activation plot, i.e., the plot of $\log$ (frequency) versus reciprocal loss-peak temperature for the $\alpha$ process. As is seen in this figure, the curve conforms to the Vogel-Tamman equation given by, ${ }^{20,21}$

$$
\log f=A-B /\left(T-T_{0}\right)
$$

where $f$ denotes frequency and $A, B$, and $T_{0}$ are parameters. The quantity $2.303 R B$ corresponds to the activation energy at high temperature, and it may be equated to the energy barrier for internal rotations of the main chain. ${ }^{13}$ On the other hand, $A$ and $T_{0}$ may be correlated with the $\log$ (frequency) of thermal vibration and that of inter-molecular interaction, respecively. ${ }^{13} \mathrm{~A}$ comparison of these parameters of the solutions of PMMA-S and

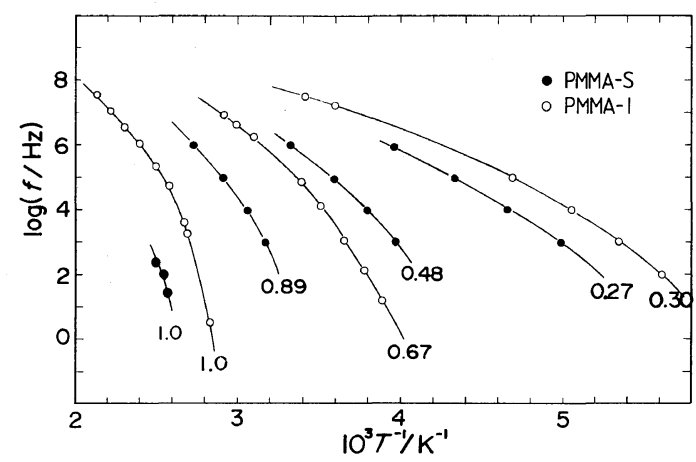

Figure 5. Arrhenius plots for the $\alpha$ process. Weight fractions of the PMMAs are given in the figure. 


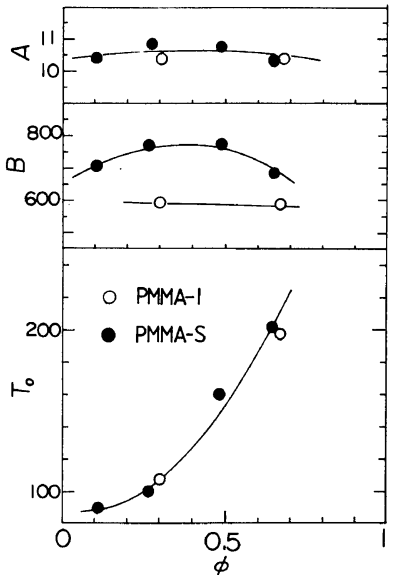

Figure 6. Parameters $A, B$, and $T_{0}$ in the VogelTamman equation versus weight fraction $\phi$ of the PMMAs.

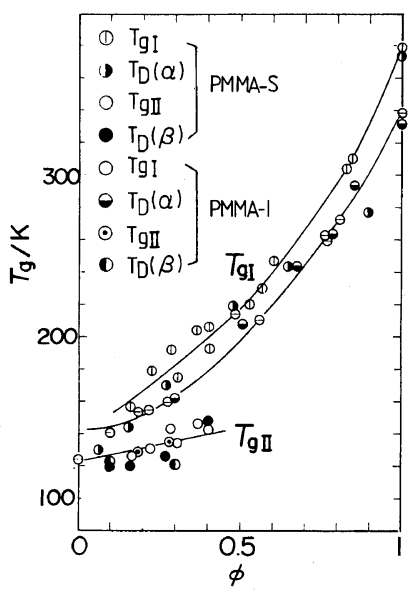

Figure 7. Concentration dependence of glass transition points $T_{\mathrm{gl}}$ and $T_{\mathrm{gll}}$ and dielectric glass transition temperatures for the $\alpha$ process $T_{\mathrm{D}}(\alpha)$ and for the $\beta$ process $T_{\mathrm{D}}(\beta)$.

PMMA-I is made in Figure 6. We did not determine these parameters for solutions with concentrations above $70 \%$, since for such solutions the $\alpha$ peak overlaps the $\beta^{\prime}$ peak exhibiting an Arrhenius type temperature dependence, and hence the feature of the primary process is obscured. As seen in Figure 6, the stereoregularity has an effect on $B$, but there is no appreciable difference in the lattér two parameters, $A$ and $T_{0}$.

Figure 7 shows the concentration dependence of glass transition temperatures determined by DTA

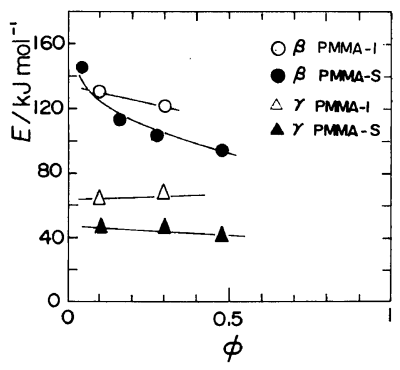

Figure 8. Activation energies $E$ for the $\beta$ and $\gamma$ processes. $\phi$ denotes weight fraction of the PMMAs.

and those by dielectric loss measurements. In the latter, this transition temperature is defined as the temperature at which $\varepsilon^{\prime \prime}$ at $0.01 \mathrm{~Hz}$ exhibits a maximum. Two glass transition points were recognized in DTA diagrams for solutions with concentrations below $40 \%$. We have designated these temperatures as $T_{\mathrm{gI}}$ and $T_{\mathrm{gII}}$ in the order of decreasing temperature. As seen in Figure 7, the dielectric glass transition temperatures for the $\alpha$ and $\beta$ processes, $T_{\mathrm{D}}(\alpha)$ and $T_{\mathrm{D}}(\beta)$, coincide with $T_{\mathrm{gl}}$ and $T_{\mathrm{gll}}$, respectively. Because the glass transition temperature is generally regarded as the temperature at which the correlation time for molecular motion becomes an order of $100 \mathrm{~s}$, or the relaxation frequency becomes $0.01 \mathrm{~Hz}$, we may conclude that $T_{\mathrm{gl}}$ is the glass transition point at which the segmental motion of PMMA molecules is frozen-in and that $T_{\text {gII }}$ is the temperature at which the rotational motion of toluene molecules is frozen-in.

The activation energies for the $\beta$ and $\gamma$ processes are shown in Figure 8 . The former decreases with concentration, while the latter is almost independent of concentration. This behavior resembles that of the system of poly(vinyl acetate)/toluene (PVAc/TOL). ${ }^{15}$ The activation energy of the $\beta$ process for PMMA solutions is about $120 \mathrm{~kJ} \mathrm{~mol}^{-1}$ at a concentration of $30 \%$ and similar to those of PS/TOL and PVAc/TOL systems at the same concentration. Therefore, it seems that the activation energy for the rotational motion of toluene molecules in polymer solutions is not much influenced by the chemical structure of polymers. On the other hand, the activation energy for the $\gamma$ process is somewhat different from one system to another. In the PMMA/TOL system, the activation energy of the PMMA-I system is higher than that of the PMMA-S system. This is consistent with the specu- 


\section{K. ADACHi and T. Kotaka}

lation that the $\gamma$ process is due to the cooperative motions of both the polymer and the solvent.

\section{REFERENCES}

1. T. G Fox, B. S. Garrett, W. E. Goode, S. Gratch, J. F. Kincaid, A. Spell, and J. D. Stroupe, J. Am. Chem. Soc., 80, 1768 (1957).

2. N. G. McCrum, B. E. Read, and G. Williams, "Anelastic and Dielectric Effects in Polymeric Solids," John Wiley, New York, 1967.

3. G. P. Mikhailov, T. I. Borisova, and D. A. Dmitrochenko, J. Tech. Phys. USSR., 26, 1924 (1956).

4. S. Havriliak, Jr., Polymer, 9, 289 (1968).

5. Y. Ishida, S. Togami, and K. Yamafuji, Kolloid Z., 222, 16 (1968).

6. J. C. Wittmann and A. J. Kovacs, J. Polym. Sci., C, No. 16, 4443 (1969).

7. Y. Iwasa, S. Mashimo, and A. Chiba, Polym. J., 8, 401 (1976).

8. A. M. North and P. J. Phillips, Trans. Faraday Soc., 63, 1537 (1967).
9. S. Mashimo, A. Chiba, and K. Shinohara, Polym. J., 6, 170 (1974).

10. P. J. Phillips and B. Singh, J. Polym. Sci., Polym. Phys. Ed., 13, 1377 (1975).

11. L. L. Burshtein and V. P. Malinovskaya, Vysokomol. Soyed. in Ser. A, 20, 428 (1978).

12. S. Mashimo, A. Chiba, and K. Shinohara, Polym. J., 8, 401 (1976).

13. K. Adachi, I. Fujihara, and Y. Ishida, J. Polym. Sci., Polym. Phys. Ed., 13, 2155 (1975).

14. K. Adachi and Y. Ishida, J. Polym. Sci., Polym. Phys. Ed., 14, 2219 (1976).

15. K. Adachi, M. Hattori, and Y. Ishida, J. Polym. Sci., Polym. Phys. Ed., 15, 693 (1977).

16. K. Adachi and Y. Ishida, Polym. J., 11, 233 (1979).

17. F. A. Bovey and G. V. D. Tiers, J. Polym. Sci., 44, 173 (1960).

18. H. Fröhlich, "Theory of Dielectrics," Clarendon Press, Oxford, 1960.

19. C. P. Smyth, "Dielectric Behavior and Structure," McGraw-Hill, New York, 1955.

20. H. Vogel, Phys. Z., 22, 645 (1921).

21. G. Tamman and W. Hesse, Z. Anorg. Allgem. Chem., 156, 245 (1926). 